\title{
Sensitive to the Digital Touch? Exploring Sensory Processing Sensitivity and Its Impact on Anthropomorphized Products in E-Commerce
}

\author{
Lingyao (Ivy) Yuan \\ Iowa State University \\ lyuan@iastate.edu
}

\author{
Jordan Barlow \\ University of St. Thomas \\ jordan.barlow@stthomas.edu
}

\begin{abstract}
Individual characteristics have a strong impact on decision-making behavior in virtual environments, including interacting with virtual agents, avatars, or animated objects. Prior IS research on individual characteristics has mostly focused on constructs such as the Big Five personality traits, emotional intelligence, and social sensitivity to understand their effects on behavior in virtual environments. However, we believe how individuals receive, process, and react to sensory information at the basic level is critical for developing perceptions that influence online behavior, including towards humanized objects through the process of anthropomorphism. This study explores sensory processing sensitivity as an inherent individual characteristic and its impact on individuals' bidding decision towards humanized products in online auctions. Results show that sensory processing sensitivity has a positive impact on perceived anthropomorphism, which in turn strongly affects willingness to pay.
\end{abstract}

\section{Introduction}

Anthropomorphism is a spontaneous automatic process that we cannot consciously control [22, 41]. When we hear and/or see an inanimate object with triggering features, though subtle, our brain automatically ascribes human form to it even though we rationally know the object is not human [24]. We know that agents like Siri and Alexa are not human, but we still anthropomorphize them while interacting with them - that is, we ascribe human characteristics and behavior to them. On the other hand, certain design flaws could also "tipoff" the user from anthropomorphizing an object and even create aversion from the users to the object. For example, a photorealistic avatar with an unsynchronized jaw movement to its voice could lead itself to the "Uncanny Valley" - a term used in anthropomorphism literature to describe the aversion people feel to nearly human objects known not to be human [43].

Anthropomorphism changes how we think and behave toward an object. When we anthropomorphize
AI agents or cars, we trust them more [14, 67]. When we anthropomorphize consumer products, we are more likely to lose self-control and consume more [30]. We are even more willing to pay more for products we anthropomorphize [70]. When we anthropomorphize instructional agents, we learn more from them [14, 4]. Thus, anthropomorphized objects have the potential to influence individuals' behavior, attitude, and perceptions $[13,32,14]$.

Anthropomorphism has practical implications in many different areas. For example, many companies are working toward making their products "smart" and more human realistic. Voice recognition assistants, such as Siri and Alexa, do not have a physical form close to a human body, but they interact with users with natural human language. Some customer support chatbots, on the other hand, do not even have voices but can provide post-sales support or technical support through written text $[5,62,61,57]$. A successful deployment of such technologies depends not only on the visual or auditory formats of the virtual agents [27], but also on whether users perceive it and interact with it as if they were interacting with a human.

The design of the object impacts the process of anthropomorphism, but design is not the only factor. Even though anthropomorphism is an automatic process and to see human in others is human nature, the activation of this process may vary from person to person. The severity of the impact may also vary. In this study, we aim to investigate the impact of personal inherent characteristics, specifically sensory processing sensitivity, on anthropomorphism.

Sensory Processing Sensitivity (SPS) is "an innate trait that is associated with individual sensitivity or responsivity to environmental and social stimuli" $[2,8]$. SPS leads people to be more prone to receive and respond to stimuli, especially social stimuli. SPS can lead to less difficulty in detecting others' feelings and an increased likelihood for emotions to be impacted by others. As a unique trait, SPS has caught the attention of psychology researchers and neuroscientists. Associations have been established between SPS and other personal traits, such as big five personality traits, social sensitivity, emotional intelligence, etc., but research on SPS is still early. In the context of virtual 
environments, the amount, intensity and frequency of delivering sensory stimuli has a much wider range and can be easily manipulated. Thus, consumers with different levels of SPS receive those stimuli differently and respond differently. Some of them can be overwhelmed while others perfectly at ease. Therefore, understanding the impact of the SPS is important in understanding how people will interact with anthropomorphized technology. Specifically, this study aims to answer the following research question:

Do people with different levels of sensory processing sensitivity behave differently towards anthropomorphized products in e-commerce?

We address this research question by studying the impact of SPS on anthropomorphism, along with the effect that ultimately has on willingness to pay in bidding behavior. We achieve this through a behavioral study and survey where participants bid on products after viewing anthropomorphized product videos. We believe understanding the impact of SPS on the process and outcomes of anthropomorphism has great potential to benefit not only academia, but also practitioners. While businesses are focusing on personal, tailored recommendations and advertisements to individual customers, personality traits tend to be overlooked or simplified. Through this study, we emphasize the role of individual sensory processing sensitivity in consumers' attitude to anthropomorphized products in online advertisements.

\section{Theory and Hypotheses}

\subsection{Anthropomorphism}

Anthropomorphism is defined as "the attribution of human form or characteristics to a non-human being" [40]. It is inherent to our nature rather than a learned skill. People start to anthropomorphize during infancy and carry it throughout their lifetime [25, 31]. Many of us have had the experience of blaming, arguing with, or occasionally threatening a machine when technology fails, just as we would do to another human. Anthropomorphism is spontaneous, pervasive, and powerful $[23,41,10]$. Even though people can mask or even correct the impact, it is impossible to control or turn off.

The underlying philosophical question of why people anthropomorphize has been the target of researchers in many fields, including psychology, sociology, and anthropology. Some people believe humans anthropomorphize to reduce the uncertainty of the unknown $[23,11,68]$ to the self. Other research argues that humans anthropomorphize to compensate for the lack of social connections with other humans [17, $18]$.
The implication of anthropomorphism in humancomputer interaction [19, 16], on the other hand, fuels the research of computer scientists, graphical designers, and engineers. The "Computers are social actors" (CASA) paradigm of computer science posits that people extend social heuristics and responses from human-to-human interaction to human-computer interactions [46, 47]. In general, the more an object looks and moves like a human, the more positive the response; however, an "Uncanny Valley" exists when an object behaves almost, or sometimes even exactly, like natural human beings. Such objects trigger a feeling of revulsion that may lead to negative responses [43, 39].

Anthropomorphism is powerful and compelling. A static image can be sufficient to trigger the automatic process of anthropomorphism [48]. However, the activation of the anthropomorphism process may not lead to an observable perception change or behavioral consequences. Combining different design factors can also increase the likelihood of activation as well as the severity of its impact. Such factors include adding human facial features, human sounds/voices, intentionality, imitation behavior, communication [15, 63], and movement [26]. Notably, the impact of anthropomorphism strengthens the emotional and behavioral responses both ways, not just positively. For instance, the design of the anthropomorphic object may lead to a more negative impact if the design is associated with an unhelpful agent [64].

All design factors need to fit holistically as a whole image rather than separated aspects. Take physical appearance and voice, for example. Users must spend more time processing information delivered by virtual agents with humanoid voices than by virtual agents with human voices. They also trust the former less than the latter [21].

To "feel" how non-human objects "feel" is critical. Making an object move at speeds similar to human speed will lead people to perceive the object with human mental states [42]. Synchronizing the voice with the movement of the object makes individuals pay more attention, be more aroused, and have a higher evaluation of the object [54].

Ample research has shown that anthropomorphism has an impact on perception, attitude and behavior [24, 54, 37, 34, 12, 70]. Prior research has argued that anthropomorphism may contribute to the evaluation of a product's value and a consumer's willingness to pay $[24,70]$. Consumers will develop more favorable attitudes towards the anthropomorphized brand than the non-anthropomorphized $[3,50]$, as long as the brand can consistently maintain its positive image [51]. Individuals perceive computers displaying flattering comments as more attractive and believable than those 
that do not display such comments [36]. Anthropomorphizing the computer interface also amplifies the positive effect of flattering comments on perceptions of the computers' performance [36].

The longer someone interacts with a virtual agent, the more likely they are to anthropomorphize it [20]. Once anthropomorphized, social connections with the objects can be established the same way with other humans. Such implied social relationships, in turn, can strengthen the impact of anthropomorphism. Individuals view the computer as significantly more positive when they are told that they are teamed with the computer to work on a task than when they are told that they are working alone using the computer [54].

The impact of individual characteristics, such as gender, culture, and personality traits, on anthropomorphism is complex [66]. Users' individual characteristics can have direct impact; for instance, females were influenced more than males by anthropomorphized agents for products with high hedonic value [65]. Individual characteristics can also have an indirect impact; for example, whether consumers can develop a humanlike social relationship with a brand mascot is moderated by individual personality [35, 33]. In addition, users also ascribe personality attributes to programs [29]. The perception of the anthropomorphized objects' characteristics combined with the users' characteristics impact users' perceptions, attitudes, and behaviors.

Biases and prejudice also apply to anthropomorphized objects [44, 60]. For instance, individuals trust computers with male voices more for technical related subjects and those with female voices for love and relationship topics [45]. Computer users not only recognize traits of dominance, submissiveness, and friendliness, but also even show favor toward a computer that exhibits similar personality traits to themselves [54]. Moreover, users prefer computers that adapt to them. The ability to adapt facilitates anthropomorphism and social presence [37]. Researchers have also shown that dominant people prefer a computer that starts out submissive but then becomes dominant, more than a computer that is consistently dominant [54].

Anthropomorphism can be triggered by multiple factors spontaneously. However, the activation of and the intensity of this process varies from person to person. Personal inherent characteristics, such as sensory processing sensitivity, would greatly impact the activation or the consequences of anthropomorphism in the virtual environment.

\subsection{Sensory Processing Sensitivity}

Sensory Processing Sensitivity (SPS), also called environmental sensitivity, is "a basic temperament/personality trait categorized by sensitivity to both internal and external stimuli, including social and emotional cues" [1, p. 1]. In providing background on SPS, we explain both parts of the term: sensory processing and sensitivity. First, sensory processing refers to an emphasis on how the brain processes sensory information received by sensory organs. Sensory information is a broad term including touch, hear, see, taste, smell, as well as social cues. Second, sensitivity refers to the levels of arousal and responsiveness, both positive and negative, in a person's brain upon receiving the sensory information. For example, a tag on the collar of a shirt may bother people with higher levels of SPS. Entering a room full of strangers is overwhelming for people high in SPS but has less impact on those with less SPS. Differences in the way the brain processes sensory (including social) cues leads to differences in behavior, judgment, and perception. Prior research also found awareness, empathy, and responsiveness are fundamental features of SPS [2].

SPS is believed to be innate and hereditary [7, 38]. It can be found not only in humans but also in over 100 other species $[69,8]$. High SPS means more sensory information in more vibrant colors flooded into the brain. Small details may "jump out". The brain will need more time to sort through the information to reach a decision. More parts of the brain get involved to process the sensory information and deeper processing of the information is activated. Therefore, people with higher SPS tend to be more attentive to details, have great depth of processing, be more cognizant of subtleties in the environment, and are capable of making better long term judgments [6, 8, 7]. Some researchers believe such a trait is genetically advantageous. However, this trait also makes people feel easily overstimulated, have high arousal responses, become easily exhausted from bearing too much information, and overload their nervous system. Some researchers argue people with high SPS are more prone to depression, though these findings are inconclusive [7]. Though some prior research has tended to focus on people with significantly higher SPS, the reality is that SPS exists on a continuum. In this study, we do not categorize individuals as "high" or "low" in SPS; rather, we measure SPS on a continuous scale.

Even though SPS is a temperament trait on its own, SPS can be found to have close association with other personality traits. Individuals with high levels of SPS tend to appear as being shy or timid; prior research has demonstrated a strong link of introversion with SPS, depending on the environment in which individuals are 
raised [7]. SPS is also positively correlated with neuroticism [7].

People with a high level of SPS may experience high sensitivity to environmental cues and even hyperor hypo-responsiveness to stimuli. This does not mean that people with a high level of SPS are cognitively atypical or suffer from neurological disorders. People with high SPS do not bare structural differences in their brain from others. Combining multiple fMRI studies of clinical disorders, Acevedo, et al. [1] found that SPS is fundamentally different from clinical disorders of Autism Spectrum Disorder (ASD), Schizophrenia, and Post-Traumatic Stress Disorder.

Prior research on the impact of SPS in the automatic process of anthropomorphism is still limited yet promising. We believe consumers with higher SPS would activate the anthropomorphism process differently from those with lower levels of SPS. Such difference will generate a behavioral difference in the context of electronic commerce.

\subsection{Research Hypotheses}

As discussed above, people with high SPS have higher awareness of the environment, are more empathetic towards others, and are more responsive to cues [2]. A non-human object designed with human characteristics carries a large amount of sensory information. Such information stands out to people with SPS and thus is more likely to trigger anthropomorphism. Once being anthropomorphized, people with high SPS will not only be more likely to be influenced by the object and more acceptable to the information delivered by the object, but also have a higher likelihood to develop similar emotions as the product, forming a close relationship. Thus, we hypothesize that:

H1. Individuals' sensory processing sensitivity level is positively related to their perceived anthropomorphism towards anthropomorphized products.

Consumers' willingness to pay is the result of cognitive processing. Prior research has found an anthropomorphic product display affects individual's willingness to pay [70]. Several different theories have been used to explain this association. Humans are more likely to perceive anthropomorphic products as similar as humans, and thus, will pay more attention to them than to non-anthropomorphic products [49]. Greater attention leads to more positive information delivered to the brain to form willingness to pay [49, 70]. Once anthropomorphized, consumers are more likely to develop human relationships towards the product, such as attachment $[56,58,70]$. Product attachment leads to the tendency to obtain ownership of the product for the purpose of sustaining this close relationship, resulting in higher willingness to pay $[59,58,70]$. Consistent with prior research, we hypothesize:

H2. Perceived anthropomorphism towards anthropomorphized products is positively related to individual's willingness to pay in online auctions.

The impact of SPS on willingness to pay is fully dependent on the activation of the anthropomorphism process. No research on SPS indicates that higher levels of SPS of itself would lead to variation in purchasing behaviors directly. The impact of SPS on purchase decisions only exists when anthropomorphism has been activated. Thus, we hypothesize:

H3. Perceived anthropomorphism towards anthropomorphized products fully mediates the relationship between sensory processing sensitivity and individuals' willingness to pay in online auction.

\section{Research Methodology}

\subsection{Participants}

We conducted two studies using a survey methodology to test our hypotheses. We contracted with Qualtrics, a survey research company, to recruit a panel of research participants from the general population of the U.S. To maintain data quality, we used six attention check questions as filters. Participants who missed more than 1 attention check were excluded from the analysis.

For Study 1, 517 participants were recruited. 62 participants failed more than 1 attention check and thus were removed, resulting in a total sample of 455 .

For Study 2, 524 participants were recruited. 26 participants were excluded, resulting in a total sample of 498.

\subsection{Procedures}

Participants viewed one (Study 1) or more (Study 2) marketing videos with anthropomorphized representations of products and were asked to provide a bid amount for how much they would be willing to pay for the product in the video. After completing the bid(s), participants were then directed to complete the remainder of the survey, which asked questions about participant perceptions of the product as well as questions about individual characteristics.

\subsection{Design}

3.3.1. Study 1 Design. Study 1 used a motion video that advertised a tablet computer. Four versions of the video were created; the four versions differed on which 
anthropomorphized features were included in the advertisement: visual/auditory, no-visual/auditory, visual/no-auditory, and no-visual/no-auditory. Each participant viewed only one (randomly assigned) version of the video. We included the possibility to see any of the versions to increase the amount of variance in the study regarding perceptions of anthropomorphism.

The visual and auditory anthropomorphism present in some versions of the video were adapted from prior research [70]. The visual appearance of the tablet in the anthropomorphized version was created through adding cartoon, human-like physical characteristics (such as eyes, nose, mouth, arms, and legs), and human-like movements (such as talking, waving, clapping, jumping, turning) to the tablet in the video. For the no-visual version, a product slide show was presented to the subject. The slide show presented different still images of the tablet.

For the auditory anthropomorphism versions, a human voice was recorded by a professional male voice actor following a written script. The voices in the auditory versions were recorded as first person so that the tablet has direct communication with the viewers. For the no-auditory versions, the script was presented in text subtitles below the video.

3.3.2. Study 2 Design. Study 2 follows a repeated measure within-subject design. Each participant viewed four videos, with each video showing a different product (i.e., TV, tablet, laptop, and camera). For each product, we created two versions of the video-one anthropomorphized and one without anthropomorphism. The anthropomorphized version contained both visual and auditory elements as described above in Study 1 [70]. Each participant was randomly assigned to see two anthropomorphized product videos and two non-anthropomorphized product videos. The order of the products and video versions received by the participants were also randomized. Thus, each participant saw a variety of products to bid on with a variety of anthropomorphism embedded.

Importantly, neither study directly tests the effects of the various versions of the videos; rather, we provide variance in the product videos seen by participants and then measure their level of perceived anthropomorphism so that we can test how the SPS of individuals affected the extent to which they anthropomorphized the products in the videos.

\subsection{Measurements}

To measure Sensory Processing Sensitivity (SPS), we adopted the 27 item highly sensitive person scale [8], the standard measure of SPS in adults. Cronbach's alpha for SPS was 0.931 in Study 1 and 0.934 in Study 2. Perceived Anthropomorphism (PA) was measured with 8 items that were adopted from prior research [55]. Cronbach's alpha for PA was 0.932 in Study 1 and 0.963 in Study 2. Both constructs were measured through items using 7-item Likert scales.

Willingness to pay (WTP) refers to the dollar amount an individual would pay to obtain possession of a product. It was measured directly after participants received all product information from the video.

Four control variables-income, age, language (whether English is the first language), and genderwere included in the research model. We also controlled for product type in Study 2.

\section{Analysis and Results}

\subsection{Analysis Strategy}

Following the guidelines of Baron and Kenny [9] to test models that contain mediation, we have tested three models for both studies. Model 1 tests the direct impact of sensory processing sensitivity on perceived anthropomorphism $(\mathrm{H} 1, \mathrm{H} 3)$. Model 2 tests the direct impact of sensory processing sensitivity on willingness to pay in order to check if there is any direct effect being mediated (H3). Model 3 tests the impact of both perceived anthropomorphism and sensory processing sensitivity on willingness to pay $(\mathrm{H} 2, \mathrm{H} 3)$. Table 1 summarizes the three models.

\section{Table 1. Models Summary}

\begin{tabular}{|l|l|}
\hline Model 1 & SPS $\rightarrow$ Perceived Anthropomorphism \\
\hline Model 2 & SPS $\rightarrow$ Willingness to Pay \\
\hline Model 3 & $\begin{array}{l}\text { SPS, Anthropomorphism } \rightarrow \text { Willingness to } \\
\text { Pay }\end{array}$ \\
\hline
\end{tabular}

\subsection{Study 1 Results}

We used standard multiple linear regression to analyze the impact of sensory processing sensitivity on perceived anthropomorphism and willingness to pay.

Table 2 presents the results. In Model 1, SPS was significantly related to the level of anthropomorphism that a participant perceived in the tablet product shown in the video. This provides support for H1. In Model 3, PA was significantly related to a participant's bid, providing support for $\mathrm{H} 2$. To test the mediation effect (H3), we compare the three models, following the guidelines of Baron and Kenny [9]. SPS has a significant effect on PA (Model 1), and in turn PA has a significant effect on Bid while controlling for effects of SPS (Model 3). However, SPS has no direct effect on Bid with or without controlling for PA (Models 2, 3). Thus, we conclude that there is no direct effect to be mediated; SPS has no direct effect on willingness to pay 
outside of its impact on perceptions of anthropomorphism, which in turn affect willingness to pay.

\begin{tabular}{|l|c|c|c|}
\hline \multicolumn{5}{|c|}{ Table 2. Regression Results } \\
\hline & Model 1 & Model 2 & Model 3 \\
\hline \multicolumn{1}{|c|}{$\boldsymbol{D V}:$} & $\boldsymbol{P A}$ & $\boldsymbol{W T P}$ & $\boldsymbol{W T P}$ \\
\hline SPS & $0.288^{* * *}$ & 72.500 & 33.600 \\
\hline PA & - & - & $13.820 *$ \\
\hline Income & 0.037 & $20.830 * * *$ & $20.320 * * *$ \\
\hline Age & $-0.010 *$ & 0.682 & 0.823 \\
\hline Language & 0.219 & 28.300 & 25.200 \\
\hline Gender & -0.160 & -16.900 & -14.700 \\
\hline *p<0.05**p<0.01 ***p<0.001
\end{tabular}

\subsection{Study 2 Results}

Study 2 follows a repeated measure design where each participant viewed four different products. Hierarchical Linear Modeling (HLM) [28, 52] was used to analyze the data, using HLM version 8.00 [53]. HLM is a form of regression that considers multiple levels of analysis in one statistical equation and can be used to control for person-level effects in a repeated-measure study. The lowest level (level 1) of the HLM model is the product bid level, which considers the bid and perceived anthropomorphism for each product; the second level (level 2) is the participant level, which accounts for individual characteristics including sensory processing sensitivity and control variables (gender, language age, and income).

Table 3 presents the results, showing least squares estimates with p-values based on robust standard errors. In Model 1, SPS was again significantly related to the level of anthropomorphism that a participant perceived in the product. This provides additional support for $\mathrm{H} 1$. In Model 3, PA was significantly related to a participant's bid, providing additional support for $\mathrm{H} 2$. For H3, SPS has a significant effect on PA (Model 1), and in turn PA has a significant effect on Bid while controlling for effects of SPS (Model 3). However, SPS has no direct effect on Bid with or without controlling for PA (Models 2, 3). Thus, as in Study 1, we conclude that there is no direct effect to be mediated. In both studies, a full mediation analysis (e.g., with bootstrapping) is not appropriate given the lack of direct effect. We cannot test if an effect between SPS and WTP is mediated because there is no effect.

The only significant difference in results between Study 1 and Study 2 is that gender was a significant predictor of bid amount in Study 2, but not in Study 1 . Specifically, females were more likely to bid higher than males in Study 2. In both studies, age was a significant predictor of perceived anthropomorphism, with effects being stronger in younger participants. In both studies, income was also a significant predictor of bid. Those who have higher incomes are likely to make higher bids on products.

\begin{tabular}{|l|c|c|c|}
\hline \multicolumn{4}{|c|}{ Table 3. HLM Results } \\
\hline $\begin{array}{l}\text { Level 1 } \\
\text { Level 2 }\end{array}$ & Model 1 & Model 2 & Model 3 \\
\hline \multicolumn{1}{|c|}{$\boldsymbol{D V}:$} & $\boldsymbol{P A}$ & $\boldsymbol{W T P}$ & $\boldsymbol{W T P}$ \\
\hline Intercept & & & \\
\hline Intercept & $3.727 * * *$ & $392.924 * * *$ & $277.720 *$ \\
\hline SPS & $0.193 * *$ & -16.530 & -22.487 \\
\hline Income & -0.037 & $65.243 * * *$ & $66.380 * * *$ \\
\hline Age & $-0.010 *$ & 0.725 & 1.025 \\
\hline Language & -0.398 & -36.888 & -24.592 \\
\hline Gender & -0.097 & $70.245^{*}$ & $73.244 *$ \\
\hline PA & - & - & $30.910 * * *$ \\
\hline TV & 0.0395 & $85.477 * * *$ & $84.258^{* * *}$ \\
\hline Tablet & 0.0350 & $-232.140 * * *$ & $-233.330 * * *$ \\
\hline Camera & -0.0372 & $-237.225 * * *$ & $-236.075 * * *$ \\
\hline
\end{tabular}

$* p<0.05 * * p<0.01 * * * p<0.001$

\section{Discussion}

\subsection{Interpretation of findings}

$\mathrm{H} 1$ and $\mathrm{H} 2$ were supported in both studies; H3 could not be tested due to an overall lack of effect between SPS and willingness to pay. Study 1 examined users providing a bid on a single product. This study was designed with a focus on providing a wide variety of manipulated anthropomorphism cues to the subject pool. Study 2 examined users providing bids on multiple products. This study was designed with a focus on examining the effects regardless of product and allowing users to see different levels of anthropomorphism in various products. Taken together, the studies provide strong evidence that (1) SPS increases the level to which users anthropomorphize products; (2) anthropomorphism affects the bidding behavior of users; and (3) SPS does not directly affect bidding behavior outside of its effects on perceived anthropomorphism. We now interpret each of these three results.

First, the results of our studies indicate that users higher in sensory processing sensitivity are more likely to anthropomorphize products. The anthropomorphized versions of the video in this study include adding visual and auditory cues to the non-human object. Those cues are designed to induce the process of anthropomorphism and exist as stimuli in the task environment. Participants with high levels of SPS are more prone to receiving those environmental stimuli and being influenced by those cues $[1,7]$. Therefore, highly sensitive people are more likely to anthropomorphize the products.

Second, the results of both studies provide evidence that users who perceived themselves to have anthropomorphized the products in the video(s) will be 
more likely to make higher bids on such products. The more participants perceive the objects as human, the more strongly they feel attachment to the product, much like they would feel attachment to another human. This attachment leads to the willingness to pay more for the product to sustain the closeness to the product [70]. This finding is consistent with prior research [70].

Third, SPS does not have a direct impact on participants' willingness to pay. As we theorized, a personal characteristic or personality trait does not have a powerful impact on willingness to pay, meaning highly sensitive people are not willing to pay more for any product without activating anthropomorphism. They are prone to the environmental cues and are easier to be influenced by those cues, such as the visual and auditory cues in this study, and the outcome of the anthropomorphism process is the higher willingness to pay for the product. This finding is consistent with prior research in that SPS captures sensitivity in receving sensory informaiton and cannot direct behavioral outcomes without involving the critial cognitive processes in between $[7,8]$.

\subsection{Limitations}

As with all research, these studies have limitations that should be considered when interpreting our results. First, users bid on the products using a Qualtrics form rather than a live auction site. The form was different in appearance than a real bidding site, and participants could only view and bid on one product one product at a time without "shopping around". Future research could replicate our study in a true bidding environment.

Second, the construct of anthropomorphism was measured using a self-report survey adopted from prior research. Anthropomorphism is a cognitive process that is automatic and spontaneous [25, 31]. It can happen prior to a human's conscious realization and awareness. Even though the items have been widely used in academic research, answering survey questions unavoidably activates the conscious processing from the participants. Therefore, the survey method does not provide direct observations to the automatic process of anthropomorphism. Researchers can use different methodologies, such as fMRI, to directly observe how anthropomorphism is triggered differently in highly sensitive people than in non-sensitive individuals.

Third, we have used four technology related products - tablet, laptop, TV, and digital camera, in both studies. Therefore, our results may not be generalized to other products, especially to hedonic products, experience products, or products belonging to non-technology related categories. Affinity to technology could be another unmeasured factor that could also play a role in bidding behavior in this context.

\subsection{Implications for future research}

Despite the above limitations, we believe the results of our studies have important theoretical contributions. Research on SPS is still young; the concept of Sensory Processing Sensitivity was not proposed until 1997. As a unique inherent personal characteristic, it acknowledges how differently we receive and respond to environmental cues. SPS has great potential for theoretical implications in multiple disciplines, especially in Information Systems (IS) where we use technology to create artificial cues in the virtual and digital environment. Our study is the first study to look at the behavioral consequences of SPS in the Information System discipline. Through this study, we would like to start the conversation on SPS and hope more researchers would be interested in this topic. The impact of SPS on other constructs and processes, such as trust, should be further studied. We present here some ideas for future research that can build on this new and important area.

First, our results suggest that people with higher levels of SPS are more likely to anthropomorphize products with visual and auditory design in the virtual environment. We utilized multiple design factors to maximize the possibility to invoke this automatic process. More research can be done to examine the impact of individual design factors. In this study, we created a cartoon-like character to avoid approaching the "uncanny valley". Would a more human realistic character have a different impact?

Next, in this study, we focus on the positive effect of SPS on anthropomorphism and willingness to pay. However, higher levels of SPS can potentially lead to negative feelings towards certain stimuli. These negative effects could potentially be overwhelming. Salient cues can have longer lasting effects or more severe impact on highly sensitive people. Those with higher levels of SPS may respond positively or negatively to flaws in the design. Future research should examine whether the "Uncanny Valley" may be "steeper" depending on SPS.

Finally, our study has great potential implications for AI design and human-computer interactions in general. Our results emphasize the role of anthropomorphism between SPS and willingness to pay. People with high levels of SPS will not bid more for a product unless they have viewed and treated the product as real humans. Personality traits and their behavioral consequences are quite complex, involving multiple underlying cognitive processes, either conscious or nonconscious. There are many undiscovered cognitive processes through which we can better discover and 
explain the impact of personal traits and characteristics on ultimate behavior.

\subsection{Implications for practice}

The findings of our study have several practical implications as well. First, our study points out to firms that individual traits such as SPS are important factors to consider when they are trying to sell products online. Spending more money on creating an anthropomorphized display of products may yield different results to customers with different levels of SPS. Customers with high SPS are more likely to be influenced by the design. Targeting those customers specifically would yield a higher return than customers with lower levels of SPS. (On the other hand, adding too much stimuli to the display could have the possibility to overwhelm the highly sensitive customer.)

Second, our research suggests that consumers should be mindful of their SPS level. Although the virtual environment creates endless possibilities to alter or even distort our reality, those refinements can be used to manipulate behaviors and perceptions of the users, some more than others. For highly sensitive people, those refinements can even be too overwhelming.

\section{Conclusion}

Although with great potential, the study of Sensory Processing Sensitivity (SPS) is new to the Information Systems discipline. In this study, we bridge SPS with anthropomorphism and observe their impact on individuals' willingness to pay in online auction. We found that people with high levels of SPS are more like to anthropomorphize a product than those with low levels of SPS. High SPS will not lead to higher willingness to pay unless the person has anthropomorphized the product.

\section{References}

[1] B. Acevedo, E. Aron, S. Pospos and D. Jessen, "The functional highly sensitive brain: a review of the brain circuits underlying sensory processing sensitivity and seemingly related disorders", Philosophical Transactions of the Royal Society B: Biological Sciences, 373 (2018), pp. 20170161.

[2] B. P. Acevedo, E. N. Aron, A. Aron, M. D. Sangster, N. Collins and L. L. Brown, "The highly sensitive brain: an fMRI study of sensory processing sensitivity and response to others' emotions", Brain and behavior, 4 (2014), pp. 580-594.

[3] P. Aggarwal and A. L. Mcgill, "When Brands Seem Human, Do Humans Act Like Brands? Automatic
Behavioral Priming Effects of Brand Anthropomorphism", Journal of Consumer Research, 39 (2012), pp. 307-323.

[4] A. Ahmadi, R. Sahragard and H. Babaie Shalmani, "Anthropomorphism-matters or not? On agent modality and its implications for teaching English idioms and design decisions", Computer Assisted Language Learning, 30 (2017), pp. 149-172.

[5] T. Araujo, "Living up to the chatbot hype: The influence of anthropomorphic design cues and communicative agency framing on conversational agent and company perceptions", Computers in Human Behavior, 85 (2018), pp. 183-189.

[6] A. Aron, S. Ketay, T. Hedden, E. N. Aron, H. Rose Markus and J. D. Gabrieli, "Temperament trait of sensory processing sensitivity moderates cultural differences in neural response", Social cognitive and affective neuroscience, 5 (2010), pp. 219-226.

[7] E. Aron, The highly sensitive person, Kensington Publishing Corp., 2013.

[8] E. N. Aron and A. Aron, "Sensory-processing sensitivity and its relation to introversion and emotionality", Journal of personality and social psychology, 73 (1997), pp. 345.

[9] R. M. Baron and D. A. Kenny, "The moderatormediator variable distinction in social psychological research: Conceptual, strategic, and statistical considerations", Journal of personality and social psychology, 51 (1986), pp. 1173.

[10] G. M. Burghardt, "Amending Tinbergen: A fifth aim for ethology", (1997).

[11] L. R. Caporael and C. M. Heyes, "Why anthropomorphize? Folk psychology and other stories", Anthropomorphism, anecdotes, and animals (1997), pp. 59-73.

[12] T. L. Chartrand, G. M. Fitzsimons and G. J.

Fitzsimons, "Automatic effects of anthropomorphized objects on behavior", Social Cognition, 26 (2008), pp. 198-209.

[13] K. E. Culley and P. Madhavan, "A note of caution regarding anthropomorphism in HCI agents", Computers in Human Behavior, 29 (2013), pp. 577 579.

[14] E. J. de Visser, S. S. Monfort, K. Goodyear, L. Lu, M. O’Hara, M. R. Lee, R. Parasuraman and F. Krueger, "A Little Anthropomorphism Goes a Long Way: Effects of Oxytocin on Trust, Compliance, and Team Performance With Automated Agents", Human factors, 59 (2017), pp. 116-133.

[15] D. C. Dennett, Kinds of minds: Toward an understanding of consciousness, Basic Books, 2008.

[16] P. Ekman, Darwin and facial expression: A century of research in review, Ishk, 2006.

[17] N. Epley, S. Akalis, A. Waytz and J. T. Cacioppo, "Creating social connection through inferential 
reproduction loneliness and perceived agency in gadgets, gods, and greyhounds", Psychological science, 19 (2008), pp. 114-120.

[18] N. Epley, A. Waytz, S. Akalis and J. T. Cacioppo, "When we need a human: Motivational determinants of anthropomorphism", Social cognition, 26 (2008), pp. 143-155.

[19] N. Epley, A. Waytz and J. T. Cacioppo, "On seeing human: a three-factor theory of anthropomorphism", Psychological review, 114 (2007), pp. 864.

[20] S. R. Fussell, S. Kiesler, L. D. Setlock and V. Yew, How people anthropomorphize robots, Proceedings of the 3rd ACM/IEEE international conference on Human robot interaction, ACM, 2008, pp. 145-152.

[21] L. Gong and C. Nass, "When a Talking-Face Computer Agent is Half-Human and Half-Humanoid: Human Identity and Consistency Preference", Human communication research, 33 (2007), pp. 163-193. [22] S. Guthrie, Faces in the Clouds, Oxford University Press, 2015.

[23] S. Guthrie, Faces in the Clouds, Oxford University Press, 1993.

[24] P. M. Hart, S. R. Jones and M. B. Royne, "The human lens: How anthropomorphic reasoning varies by product complexity and enhances personal value", Journal of Marketing Management, 29 (2013), pp. 105121.

[25] G. Hatano and K. Inagaki, "Young children's naive theory of biology", Cognition, 50 (1994), pp. 171-188.

[26] F. Heider and M. Simmel, "An experimental study of apparent behavior", The American Journal of Psychology, 57 (1944), pp. 243-259.

[27] R. R. Hoffman, The psychology of expertise: Cognitive research and empirical AI, Psychology Press, 2014.

[28] D. A. Hofmann, "An overview of the logic and rationale of hierarchical linear models", Journal of management, 23 (1997), pp. 723-744.

[29] T. Holtgraves, S. Ross, C. Weywadt and T. Han, "Perceiving artificial social agents", Computers in human behavior, 23 (2007), pp. 2163-2174.

[30] J. D. Hur, M. Koo and W. Hofmann, "When temptations come alive: How anthropomorphism undermines self-control", Journal of Consumer Research, 42 (2015), pp. 340-358.

[31] K. Inagaki and K. Sugiyama, "Attributing human characteristics: Developmental changes in over-and underattribution", Cognitive Development, 3 (1988), pp. $55-70$.

[32] R. Khan, S. U. Khan, R. Zaheer and S. Khan, Future internet: the internet of things architecture, possible applications and key challenges, 2012 10th international conference on frontiers of information technology, IEEE, 2012, pp. 257-260.

[33] S. Kim and A. L. McGill, "Gaming with Mr. Slot or gaming the slot machine? Power,

anthropomorphism, and risk perception", Journal of Consumer Research, 38 (2011), pp. 94-107.

[34] J. R. Landwehr, A. L. McGill and A. Herrmann, "It's got the look: The effect of friendly and aggressive "facial" expressions on product liking and sales", Journal of marketing, 75 (2011), pp. 132-146.

[35] C. D. Lanier Jr, C. S. Rader and A. R. Fowler III, "Anthropomorphism, marketing relationships, and consumption worth in the Toy Story trilogy1", Journal of Marketing Management, 29 (2013), pp. 26-47. [36] E.-J. Lee, "What triggers social responses to flattering computers? Experimental tests of anthropomorphism and mindlessness explanations", Communication Research, 37 (2010), pp. 191-214.

[37] K. M. Lee, N. Park and H. Song, "Can a robot be perceived as a developing creature?", Human communication research, 31 (2005), pp. 538-563. [38] C. L. Licht, E. L. Mortensen and G. M. Knudsen, "Association between sensory processing sensitivity and the 5-HTTLPR Short/Short genotype", Biol.

Psychiatry, 69 (2011), pp. 152S-153S.

[39] J.-L. Lugrin, J. Latt and M. E. Latoschik, Anthropomorphism and Illusion of Virtual Body Ownership, ICAT-EGVE, 2015, pp. 1-8.

[40] I. Merriam-Webster, Webster's ninth new collegiate dictionary, Merriam-Webster, 1983.

[41] S. Mithen and P. Boyer, Anthropomorphism and the evolution of cognition, JSTOR, 1996.

[42] C. K. Morewedge, J. Preston and D. M. Wegner, "Timescale bias in the attribution of mind", Journal of personality and social psychology, 93 (2007), pp. 1. [43] M. Mori, K. F. MacDorman and N. Kageki, "The uncanny valley [from the field]", IEEE Robotics \& Automation Magazine, 19 (2012), pp. 98-100. [44] C. Nass, B. Fogg and Y. Moon, "Can computers be teammates?", International Journal of HumanComputer Studies, 45 (1996), pp. 669-678.

[45] C. Nass, Y. Moon and N. Green, "Are machines gender neutral? Gender-stereotypic responses to computers with voices", Journal of applied social psychology, 27 (1997), pp. 864-876.

[46] C. Nass, J. Steuer and E. R. Tauber, Computers are social actors, Proceedings of the SIGCHI conference on Human factors in computing systems, 1994, pp. 72-78.

[47] C. I. Nass, Y. Moon and J. Morkes, "Computers Are Social Actors: A Review of Current", Human values and the design of computer technology (1997), pp. 137.

[48] K. L. Nowak, M. A. Hamilton and C. C. Hammond, "The effect of image features on judgments 
of homophily, credibility, and intention to use as avatars in future interactions", Media Psychology, 12 (2009), pp. 50-76.

[49] J. E. Plaks, S. J. Stroessner, C. S. Dweck and J. W. Sherman, "Person theories and attention allocation: Preferences for stereotypic versus counterstereotypic information", Journal of personality and social psychology, 80 (2001), pp. 876.

[50] M. Puzakova, H. Kwak and J. F. Rocereto, "Pushing the envelope of brand and personality: antecedents and moderators of anthropomorphized brands", Advances in Consumer Research, 36 (2009), pp. 413-420.

[51] M. Puzakova, H. Kwak and J. F. Rocereto, "When humanizing brands goes wrong: the detrimental effect of brand anthropomorphization amid product wrongdoings", Journal of marketing, 77 (2013), pp. 81 100.

[52] S. W. Raudenbush and A. S. Bryk, Hierarchical linear models: Applications and data analysis methods, Sage, 2002.

[53] S. W. Raudenbush, A. S. Bryk, Y. F. Cheong and R. Congdon, HLM 8 for Windows, Skokie, IL: Scientific Software International, Inc., 2019. [54] B. Reeves and C. Nass, "The Media Equation: How People Treat Computers, Television,? New Media Like Real People? Places", Computers \& Mathematics with Applications, 33 (1997), pp. 128128.

[55] M. Salem, F. Eyssel, K. Rohlfing, S. Kopp and F. Joublin, "To err is human (-like): Effects of robot gesture on perceived anthropomorphism and likability", International Journal of Social Robotics, 5 (2013), pp. 313-323.

[56] H. N. Schifferstein and E. P. Zwartkruis-Pelgrim, "Consumer-product attachment: Measurement and design implications", International journal of design, 2 (2008).

[57] R. M. Schuetzler, G. M. Grimes and J. S. Giboney, "An Investigation of Conversational Agent Relevance, Presence, and Engagement", (2018). [58] S. E. Schultz, R. E. Kleine and J. B. Kernan, "'These are a few of my favorite things': Toward an explication of attachment as a consumer behavior construct", Advances in consumer research, 16 (1989), pp. 359-366.

[59] N. Schwarz and G. L. Clore, "Mood, misattribution, and judgments of well-being: informative and directive functions of affective states", Journal of personality and social psychology, 45 (1983), pp. 513.

[60] J. Scott, "Rational choice theory", Understanding contemporary society: Theories of the present (2000), pp. 126-138.

[61] A.-M. Seeger, J. Pfeiffer and A. Heinzl, When do we need a human? Anthropomorphic design and trustworthiness of conversational agents, Proceedings of the Sixteenth Annual Pre-ICIS Workshop on HCI Research in MIS, AISeL, Seoul, Korea, 2017. [62] B. T. Sheehan, Customer service chatbots: Anthropomorphism, adoption and word of mouth, Queensland University of Technology, 2018.

[63] P. D. Tremoulet and J. Feldman, "Perception of animacy from the motion of a single object", PERCEPTION-LONDON-, 29 (2000), pp. 943-952. [64] H. C. V. Vugt, J. N. Bailenson, J. F. Hoorn and E. A. Konijn, "Effects of facial similarity on user responses to embodied agents", ACM Transactions on Computer-Human Interaction (TOCHI), 17 (2010), pp. 7.

[65] L. C. Wang, J. Baker, J. A. Wagner and K.

Wakefield, "Can a retail web site be social?", Journal of marketing, 71 (2007), pp. 143-157.

[66] A. Waytz, J. Cacioppo and N. Epley, "Who sees human? The stability and importance of individual differences in anthropomorphism", Perspectives on Psychological Science, 5 (2010), pp. 219-232.

[67] A. Waytz, J. Heafner and N. Epley, "The mind in the machine: Anthropomorphism increases trust in an autonomous vehicle", Journal of Experimental Social Psychology, 52 (2014), pp. 113-117.

[68] A. Waytz, C. K. Morewedge, N. Epley, G. Monteleone, J.-H. Gao and J. T. Cacioppo, "Making sense by making sentient: effectance motivation increases anthropomorphism", Journal of Personality and Social Psychology, 99 (2010), pp. 410.

[69] M. Wolf, G. S. Van Doorn and F. J. Weissing, "Evolutionary emergence of responsive and unresponsive personalities", Proceedings of the National Academy of Sciences, 105 (2008), pp. 15825 15830.

[70] L. Yuan and A. R. Dennis, "Acting Like Humans? Anthropomorphism and Consumer's Willingness to Pay in Electronic Commerce", Journal of Management Information Systems, 36 (2019), pp. 450-477. 